University of Wollongong

Research Online

Australian Institute for Innovative Materials -

Papers

Australian Institute for Innovative Materials

$1-1-2014$

\title{
A novel codoping approach for enhancing the performance of polypyrrole cathode in a bioelectric battery
}

\author{
Yang Yang \\ University of Wollongong, yy214@uow.edu.au \\ Caiyun Wang \\ University of Wollongong, caiyun@uow.edu.au \\ Chunming Zhang \\ National Center For Nanoscience \& Technology \\ Dan Wang \\ National Engineering Research Center for Nanotechnology, China \\ Dannong He \\ National Center For Nanoscience \& Technology
}

See next page for additional authors

Follow this and additional works at: https://ro.uow.edu.au/aiimpapers

Part of the Engineering Commons, and the Physical Sciences and Mathematics Commons

Research Online is the open access institutional repository for the University of Wollongong. For further information contact the UOW Library: research-pubs@uow.edu.au 


\title{
A novel codoping approach for enhancing the performance of polypyrrole cathode in a bioelectric battery
}

\author{
Abstract \\ A conducting polymer (CP) based bioelectric battery provides a promising alternative to commercial \\ lithium batteries to drive biomedical devices. However, the low power density limits practical application. \\ Here, we synthesize a polypyrrole (PPy)/anthraquinone sulfonate (AQS)/reduced graphene oxide (r-GO) \\ composite via a facile electrochemical route, and use this as a novel cathode material for bioelectric \\ batteries. The presence of r-GO significantly enhanced the electrochemical properties of PPy and led to \\ greatly improved cell performance compared to that of PPy/AQS. The resultant PPy/AQS/r-GO composite \\ delivered a maximum power density of $6240.5 \mathrm{~mW} \mathrm{~m}(-2), 14.2$ times higher than that of PPy/p- \\ toluenesulfonate (pTS) as reported previously.
}

\section{Keywords}

polypyrrole, performance, codoping, novel, battery, approach, bioelectric, enhancing, cathode

Disciplines

Engineering | Physical Sciences and Mathematics

\section{Publication Details}

Yang, Y., Wang, C., Zhang, C., Wang, D., He, D. \& Wallace, G. G. (2014). A novel codoping approach for enhancing the performance of polypyrrole cathode in a bioelectric battery. Carbon, 80 691-697.

\section{Authors}

Yang Yang, Caiyun Wang, Chunming Zhang, Dan Wang, Dannong He, and Gordon G. Wallace 


\title{
A Novel Codoping Approach for Enhancing the Performance of Polypyrrole
}

\section{Cathode in a Bioelectric Battery}

\author{
Yang Yang ${ }^{1,2}$, Caiyun Wang ${ }^{2}$, Chunming Zhang ${ }^{1}$, Dan Wang ${ }^{1}$, Dannong $\mathrm{He}^{1}$ and Gordon G. Wallace ${ }^{{ }^{2}}$ \\ ${ }^{1}$ National Engineering Research Center for Nanotechnology, No.28 East Jiangchuan Road, Shanghai, 200241, P.R.
}

China

${ }^{2}$ ARC Centre of Excellence for Electromaterials Science, Intelligent Polymer Research Institute, AIIM Facility, Innovation Campus, University of Wollongong, NSW 2522, Australia.

\begin{abstract}
A conducting polymer (CP) based bioelectric battery provides a promising alternative to commercial lithium batteries to drive biomedical devices. However, the low power density limits practical application. Here, we synthesize a polypyrrole (PPy)/anthraquinone sulfonate (AQS)/reduced graphene oxide (r-GO) composite via a facile electrochemical route, and use this as a novel cathode material for bioelectric batteries. The presence of r-GO significantly enhanced the electrochemical properties of PPy and led to greatly improved cell performance compared to that of PPy/AQS. The resultant PPy/AQS/r-GO composite delivered a maximum power density of $6240.5 \mathrm{~mW} \mathrm{~m}^{-2}$, 14.2 times higher than that of PPy/p-toluenesulfonate ( $p$ TS) as reported previously.
\end{abstract}

\section{Introduction}

Recently, there is growing interest in micro-batteries for biomedical applications. One strategy is to develop the bioelectric battery, which involves taking advantage of body fluid as electrolyte and selecting materials with associated reaction products that are safe for implantation into the

\footnotetext{
* Corresponding author, Tel:+86-21-34291286, Fax:+86-21-34291125. E-mail: zhangchm2003@163.com Tel.: +61 24221 3127; Fax: +61 24298 3114; E-mail: gwallace@uow.edu.au.
} 
human body. The removal of the case is essential to the miniaturization of batteries [1-4]. Conducting polymers (CPs) as air cathode in bioelectric batteries have demonstrated acceptable power density, which could be sufficient to drive some low power implantable devices such as pacemakers or biomonitoring systems [5-7].

There still remains a great potential to increase the power density of CPs based bioelectric battery for practical applications. The chemical/physical properties of polypyrrole (PPy) can be tuned by varying the dopant. An electrocatalyst for oxygen reduction reaction (ORR), anthraquinone sulfonate (AQS), has been incorporated into PPy as dopant to further improve electrocatalytic activity [8-10]. The enhanced electrocatalytic activity of the AQS/PPy composite can be due to the more favorable kinetics and electron transport environment in the presence of conducting PPy matrix and redox-active AQS [10].

However, the poor conductivity of CPs in their reduced state severely limits their performances when used in bioelectric batteries. It is reported that that the resistance of poly (3,4-ethylenedioxythiophene) (PEDOT) increases dramatically in the reduced state, resulting in a significant discharge plateau decrease in a bioelectric battery and even rendered the battery inactive [5, 11]. Therefore, improving the electrical conductivity of CPs, in particular at its reduced state is of great importance. A promising route to achieve this goal is the formation of composites with conductive carbon materials [12, 13].

Graphene, a monolayer of $\mathrm{sp}^{2}$ bonded carbon atoms, is the most recently uncovered carbon nanocomponent and has aroused great interest in energy storage [14-18]. The introduction of 
graphene into PPy is expected to greatly improve the electrochemical properties of PPy due to its high conductivity, large surface area and excellent mechanical properties. The effective integration of graphene into PPy has been achieved during electro-polymerization to form PPy/graphene oxide (GO) composite followed by a direct electrochemical reduction [19-21]. The resultant PPy/graphene composite exhibited greatly enhanced electrochemical performance in terms of rate capability and cycling stability in either supercapacitors or lithium batteries [19, 21].

In this work, we demonstrate a new co-dopants system, incorporating AQS and reduced graphene oxide (r-GO) into PPy, forming PPy/AQS/r-GO composite. The as-prepared composite allows us to achieve both enhanced electrocatalytic activity and power density. The PPy/AQS/r-GO composite displayed a high discharge voltage of $0.78 \mathrm{~V}, 320 \mathrm{mV}$ higher than that of PPy/AQS at a current density of $800 \mu \mathrm{A} \mathrm{cm}{ }^{-2}$. It also delivered a maximum power density of $6240.5 \mathrm{~mW} \mathrm{~m}^{-2}$, far higher than that of PPy/pTS (440.1 $\left.\mathrm{mW} \mathrm{m}^{-2}\right)$.

\section{Experimental}

\subsection{Materials}

Anthraquinone-2-sulfonic acid sodium salt (98\%) was purchased from Ourchem. Pyrrole and phosphate buffered saline (PBS) were obtained from Sigma-Aldrich. Toluene-4-sulphonic (sodium salt) was from Aladdin. Pyrrole was freshly distilled, while all other chemicals were used as-supplied. Graphene oxide was prepared as previously reported [21].

\subsection{Electrode fabrication}

The PPy films were galvanostatically electrodeposited on a stainless steel mesh (Hongye Stainless Steel Wire Cloth Co. Ltd.) from an aqueous solution containing the dopant and $0.1 \mathrm{M}$ pyrrole 
monomer. The concentration of sodium p-toluenesulfonate, AQS and GO used in this work was 0.1 M, 0.005 $\mathrm{M}_{\text {and }} 1 \mathrm{mg} \mathrm{ml}^{-1}$, respectively. The applied current density was $0.5 \mathrm{~mA} \mathrm{~cm}^{-2}$, and the amount of charge consumed was $1.2 \mathrm{C} \mathrm{cm}^{-2}$ for all PPy samples. PPy/AQS/r-GO film was obtained by electrochemically reducing PPy/AQS/GO film in PBS $(\mathrm{pH}=7.4)$ solution at a constant potential of $-1.1 \mathrm{~V}$ (vs SEC) for 40 minutes.

\subsection{Basic characterization}

The surface morphologies of the films were investigated using a HITACHI S-4800 field emission scanning electron microscope. Thermogravimetric analysis (TGA) was performed under a nitrogen flow $\left(90 \mathrm{ml} \mathrm{min}{ }^{-1}\right)$ at a heating rate of $20{ }^{\circ} \mathrm{C} \mathrm{min}^{-1}$, using a Linseis STAPt1600 Thermogravimetric Analyzer. The samples were dried in a vacuum oven at $60{ }^{\circ} \mathrm{C}$ overnight before the TGA test. The elemental analysis was conducted in a Microanalytical Unit (Carlo Erba 1106). The specific surface area of the sample was measured by a 3H-2000 specific surface area instrument (Beishide Instrument-ST Co., Ltd., Beijing, China) using $\mathrm{N}_{2}$ adsorption. FT-IR spectra were recorded on a FT-IR spectrometer (Nicolet 6700) over the range $650 \mathrm{~cm}^{-1}$ to $2000 \mathrm{~cm}^{-1}$.

All the electrochemical experiments were carried out in a standard three-electrode system comprising a Pt mesh counter electrode, a saturated calomel electrode (SCE), and a PPy working electrode. Cyclic voltammetry of the polymer was performed on electrochemical workstation (CHI 660D). EIS measurements were carried out using an Autolab PGSTAT302N, and the frequency range spanned from $100 \mathrm{kHz}$ to $0.01 \mathrm{~Hz}$ with an AC amplitude of $10 \mathrm{mV}$ at the applied potentials. 


\subsection{Battery Assembly and Testing}

The cells were fabricated with PPy cathodes $(1 \mathrm{~cm} \times 1 \mathrm{~cm})$ and Mg alloy (AZ61) anodes $(2 \mathrm{~cm} \times 5$ $\mathrm{cm})$ in a one component cell with $20 \mathrm{ml}$ PBS ( $\mathrm{pH}=7.4)$ solution , and tested using a battery-testing device (NEWARE BTS, Shenzhen, China).

\section{Results and discussion}

Using conditions described in the Experimental section, a coherent, adherent film on stainless steel mesh was obtained for either the single dopant or co-dopants system. The chronopotentiograms (Figure 1) both showed an initial spike followed by a slow potential decrease as polymer growth proceeded, approaching a steady potential of ca. $0.50 \mathrm{~V}$ and 0.43 V for PPy/AQS and PPy/AQS/GO, respectively. The lower deposition potential obtained for PPy/AQS/GO suggests enhanced conductivity. The reduction of GO in the PPy/AQS/GO composite was characterized by repeated scanning cyclic voltammetry over the potential range of -1.3 to $0.5 \mathrm{~V}$ (Figure 1 inset). A large cathodic current response was generated in the region of -0.8 to $-1.3 \mathrm{~V}$ in the first cycle, which could be attributed to the reduction of GO. This reduction current decreased significantly in the second cycle and became negligible after several scans, suggesting the complete reduction of GO. This result is consistent with our previous reports [21]. 


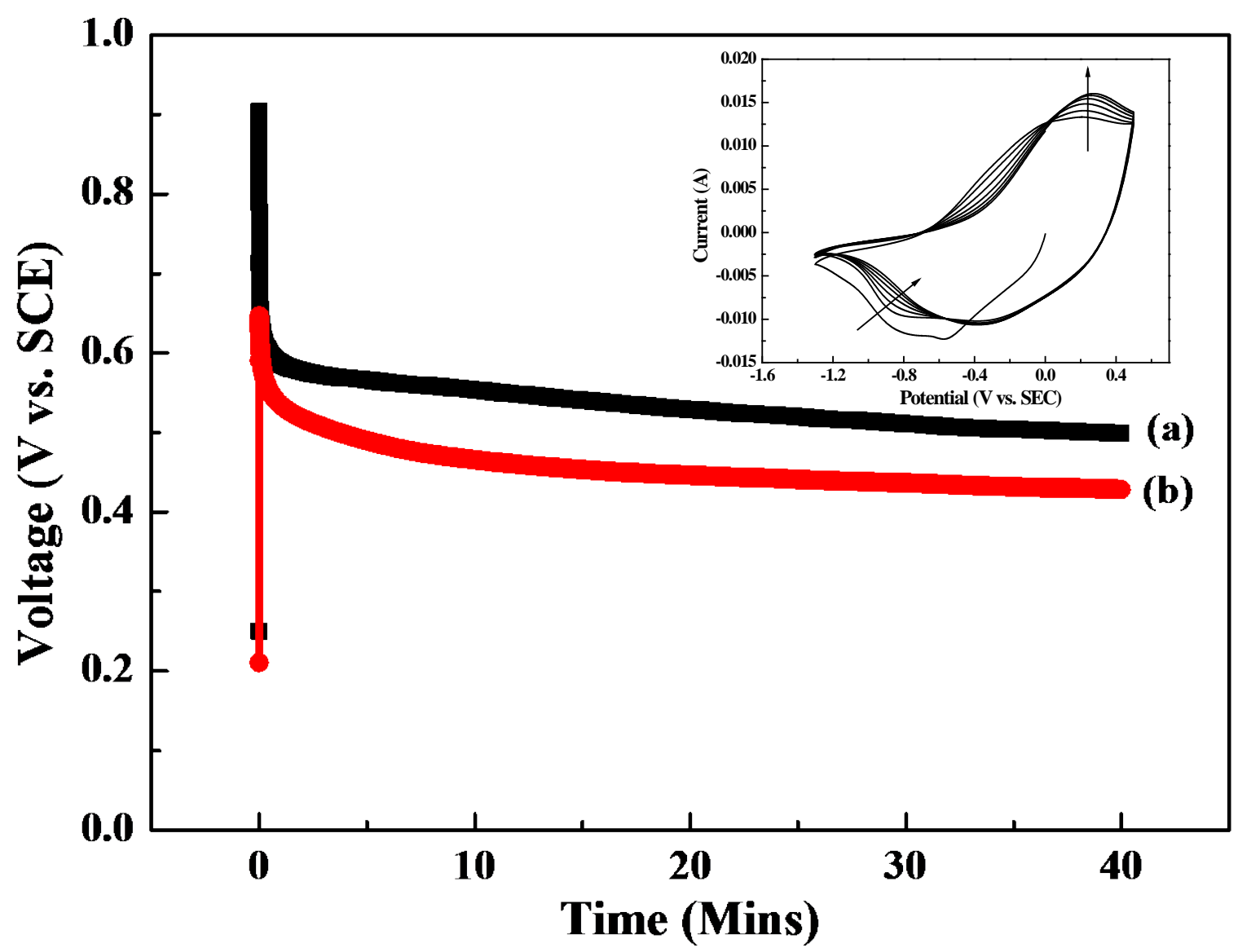

Figure 1: Chronopotentiograms of (a) PPy/AQS and (b) PPy/AQS/GO composites at a current density of $0.5 \mathrm{~mA} \mathrm{~cm}^{-2}$ for 40 minutes. Inset, cyclic voltammograms of PPy/AQS/GO in PBS $(\mathrm{pH}=7.4)$ at a scan rate of $50 \mathrm{mV} \mathrm{s}^{-1}$. Arrows direction, increasing cycling number.

The surface and cross sectional morphologies of the PPy/AQS and PPy/AQS/r-GO composites were investigated by SEM. The morphology observed for the PPy/AQS/GO was similar to PPy/AQS/r-GO and is not shown here. PPy/AQS/r-GO (Figure 2A) exhibited a porous, wrinkled structure in contrast to the typical cauliflower morphology observed for PPy/AQS (Figure 2B). The wrinkled morphology stems from the physical nature of the GO sheets that provide a template for PPy deposition, as reported in our previous study [21]. The inner structure of the PPy/AQS/r-GO composite was porous as revealed in the cross-sectional view (Figure 2C), compared to the solid, compact structure for PPy/AQS (Figure 2D). This unique porous structure ensures easy access of electrolyte and oxygen to the electrode material. The 
surface area of the as-obtained PPy/AQS/r-GO composite was measured to be $30.25 \mathrm{~m}^{2} \mathrm{~g}^{-1}$ by nitrogen adsorption/desorption method (Figure S1, ESI), which was much higher than that of PPy/AQS (4.17 $\left.\mathrm{m}^{2} \mathrm{~g}^{-1}\right)$. The larger surface area of PPy/AQS/r-GO can be due to its porous structure. Its pore size distribution profile reveals the presence of a hierarchical pore structure consisting of 4-40 nm macro/mesopores for PPy/AQS/r-GO.

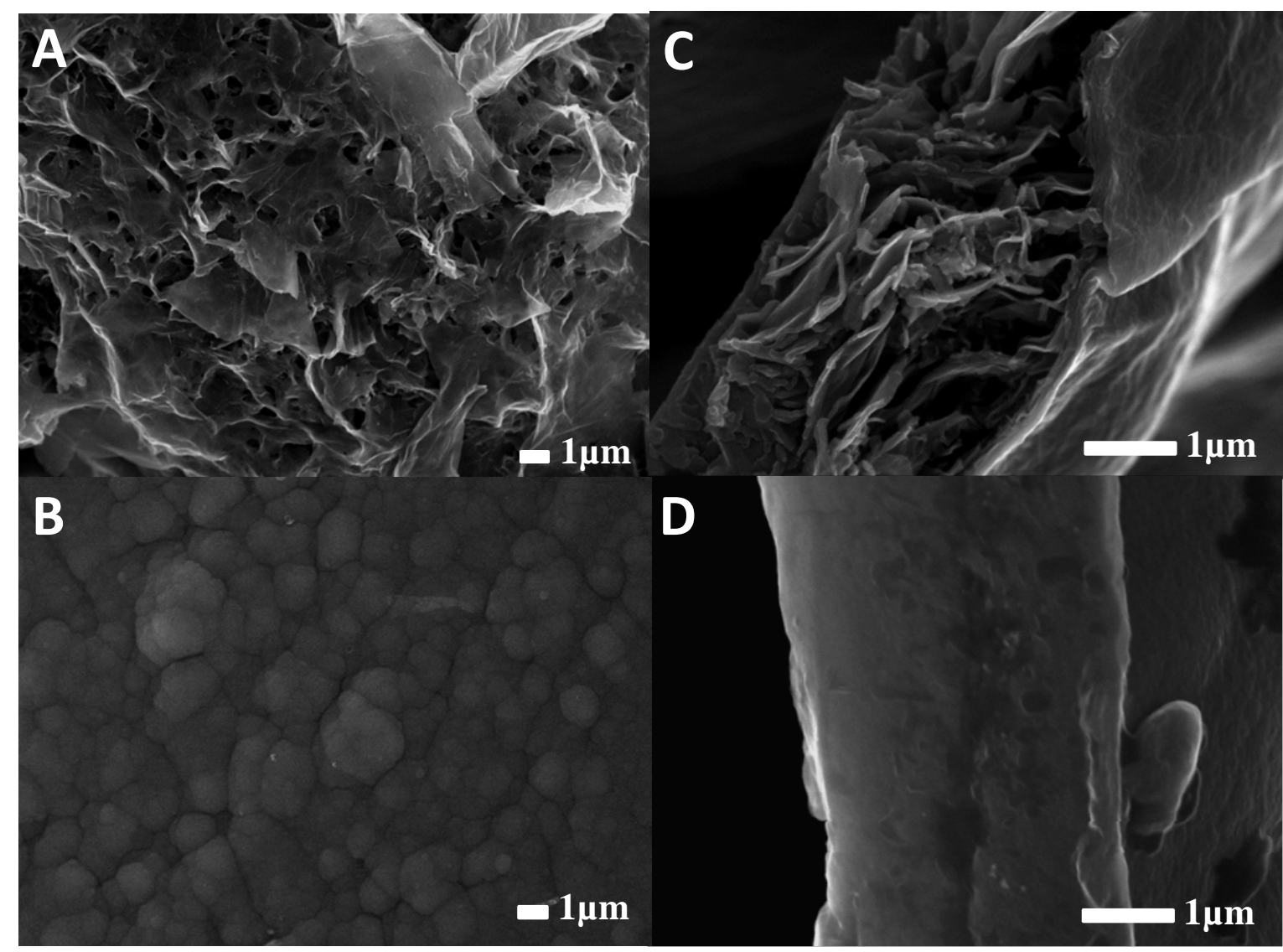

Figure 2: SEM images of (A, C) PPy/AQS and (B, D) PPy/AQS/r-GO.

The thermal stability of PPy/AQS and PPy/AQS/r-GO composites is depicted in Figure 3. A large mass loss (12.3\%) for PPy/AQS beyond $300{ }^{\circ} \mathrm{C}$ is attributed to the loss of $\mathrm{SO}_{3} \mathrm{H}$ groups $[22,23]$. In contrast, the mass loss of PPy/AQS/r-GO during this temperature range was only $6.3 \%$, which can be explained by the lower content of AQS in the composites and good thermal stability of r-GO in the matrix. The total mass loss at $300{ }^{\circ} \mathrm{C}$ was $43.1 \%$ and $38.0 \%$ for PPy/AQS and PPy/AQS/r-GO, respectively. The elemental analysis (\%) results are: PPy/AQS, C 
60.81, N 8.50, S 6.24, and H 3.51; PPy/AQS/r-GO, C 65.14, N 8.88, S 4.15 and H 3.96. Given the structure of AQS (Figure 4), the calculated weight percentage of AQS is $55.97 \%$ and $37.22 \%$ for PPy/AQS and PPy/AQS/r-GO, respectively. The low content of AQS component in the latter composite is due to the co-incorporation of the r-GO component. This composite was also examined by Fourier transformed infrared (FTIR) spectra (Figure 5). In the region from 1300 to $1250 \mathrm{~cm}^{-1}$ corresponding to $\mathrm{C}-\mathrm{H}$ or $\mathrm{C}-\mathrm{N}$ in-plane deformation modes, the peak (denoted as $*$ ) was situated at $1243 \mathrm{~cm}^{-1}$ and $1264 \mathrm{~cm}^{-1}$ for PPy/AQS and PPy/AQS/r-GO, respectively. The blue shift of this band obtained for PPy/AQS/r-GO suggests that the introduction of r-GO changes the skeletal vibrations involving the delocalized $\pi$-electrons for the polymer [24, 25].

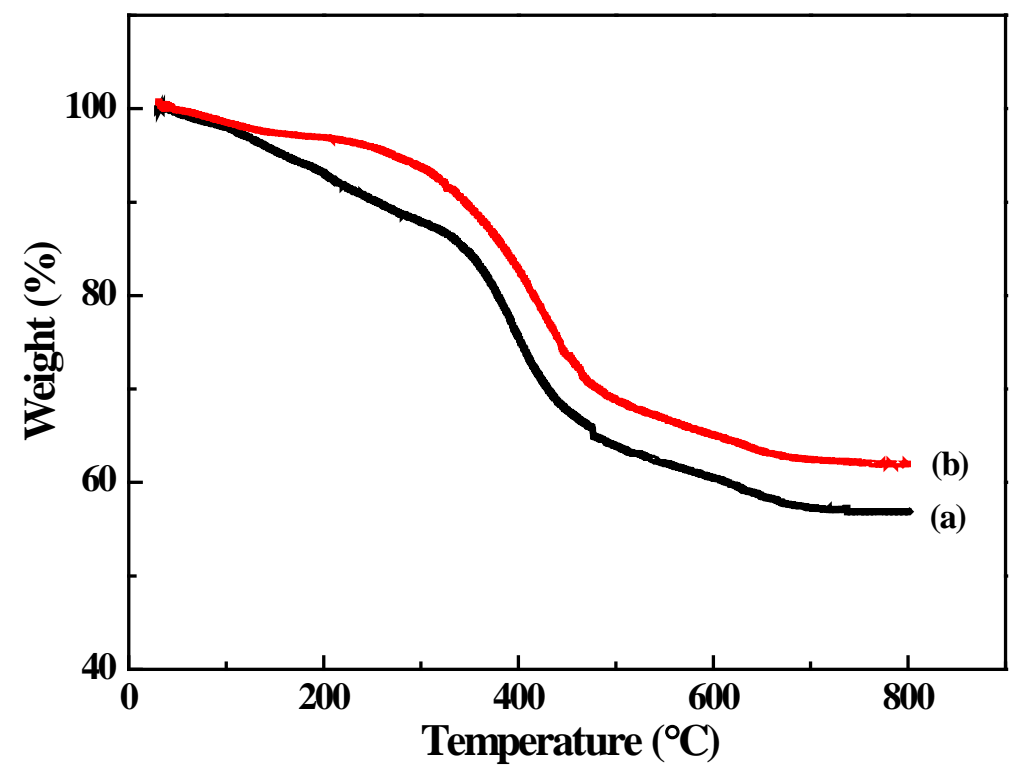

Figure 3: TGA curves of (a) PPy/AQS and (b) PPy/AQS/r-GO.<smiles>O=C1c2ccccc2C(=O)c2cc([SeH3])ccc21</smiles>

Figure 4: Schematic structure of AQS. 


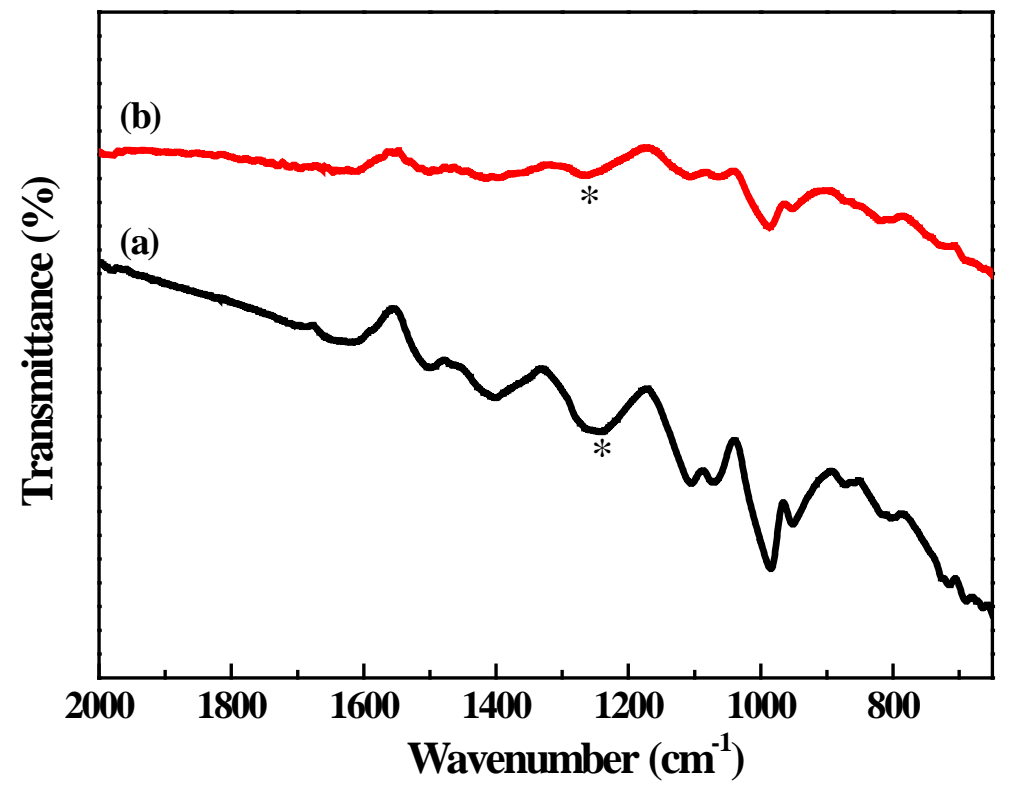

Figure 5: FTIR spectra of (a) PPy/AQS and (b) PPy/AQS/r-GO.

Cyclic voltammograms of both PPy electrodes were obtained in PBS solution (Figure 6 (A)). It can be seen that PPy/AQS presented a redox couple with the cathodic peak and anodic peak centred at approximately $-0.82 \mathrm{~V}$ and $-0.11 \mathrm{~V}$, respectively. For PPy/AQS/r-GO, the redox peaks became broader, and the potential separation of the reduction $(-0.61 \mathrm{~V})$ and oxidation peaks (-0.29 V) became smaller in comparison with PPy/AQS. Note that PPy/AQS/r-GO also exhibited higher current response. The introduction of r-GO leads to increased conductivity and a porous structure, which ensure AQS undergoes electrochemical redox reaction (Figure 7) quite efficiently within the PPy matrix, resulting in the improved electroactivity [26]. In Figure 6 (B), the CV curve recorded in $\mathrm{O}_{2}$-saturated solution only shows a broad cathodic peak for PPy/AQS at $-0.94 \mathrm{~V}$, in contrast, a more positive and pronounced cathodic peak was observed for PPy/AQS/r-GO at $-0.71 \mathrm{~V}$. This can be explained by the fact that the electronic conductivity provided by r-GO promotes the redox activity of AQS leading to an enhanced electrochemical performance even when PPy is in the reduced (less conducting) state. 
Furthermore, its porous structure also favors the electrocatalytic activity of AQS by facilitating easier access of electrolyte and oxygen. It should be also noted that graphene nanosheets defects and other oxygen-containing functional groups can also contribute to this enhanced performance due to the electrocatalytic activity for oxygen reduction as described previously [27]. However, PPy and graphene only possess a mild catalytic activity for oxygen reduction [28-29]. Our CV results (Figure S2, ESI) also confirm this conclusion, as evidenced from the slightly increased cathodic peak current in $\mathrm{O}_{2}$-saturated solution for PPy/pTS and PPy/r-GO, respectively. There is no doubt that this pronounced cathodic peak at $-0.71 \mathrm{~V}$ should be contributed mainly from AQS and the porous structure.
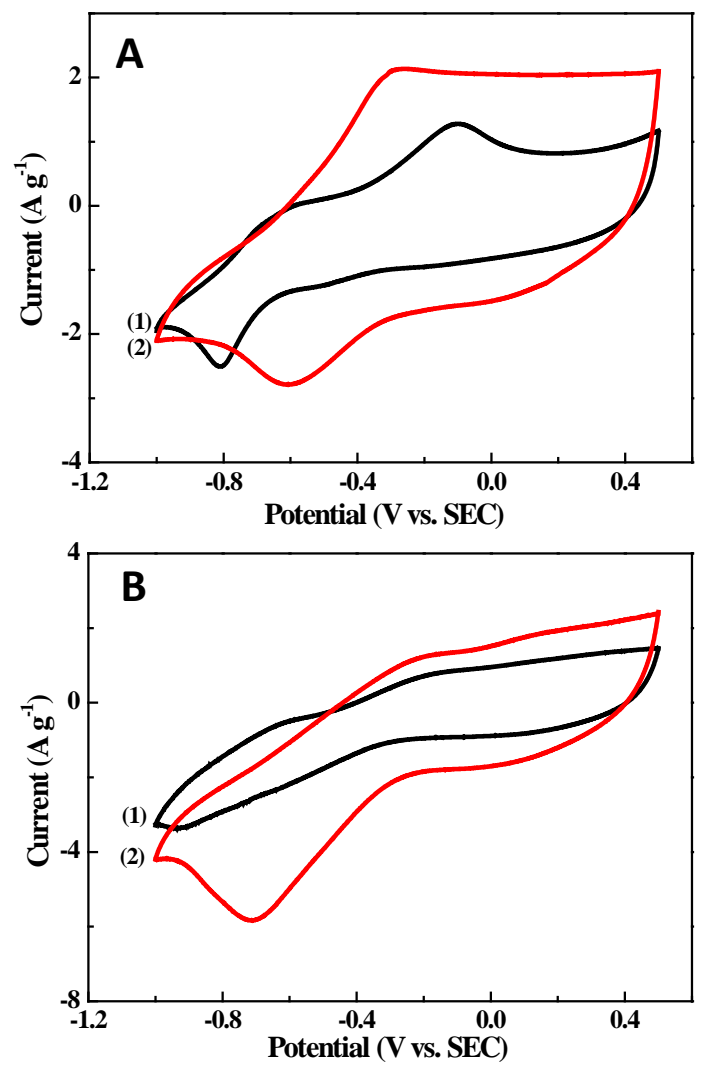

Figure 6: Cyclic voltammograms of PPy/AQS and PPy/AQS/r-GO obtained in PBS (pH=7.4) solution (A) without, (B) with $\mathrm{O}_{2}$-saturated at a scan rate of $5 \mathrm{mV} \mathrm{s}^{-1}$. (Curve 1, PPy/AQS; Curve 2, PPy/AQS/r-GO) 

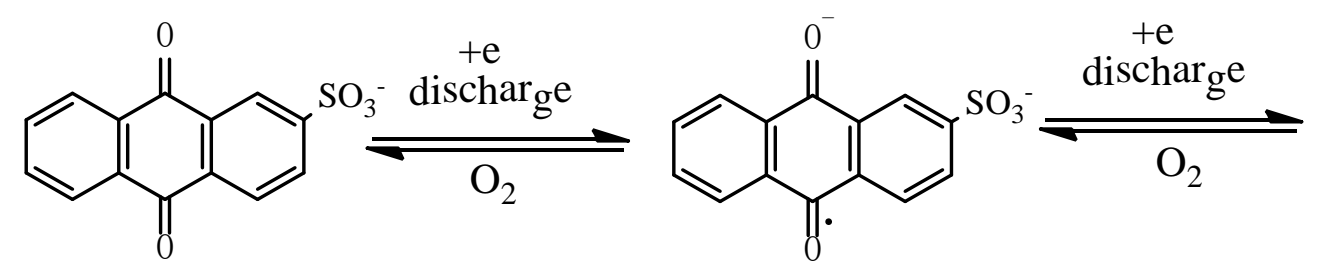<smiles>O=C1c2ccccc2C(=O)c2cc([Se])ccc21</smiles>

Figure 7: Electrochemical-chemical mechanism of AQS [10, 28].

PPy becomes reduced when it is used in a bioelectric battery, and with conductivity greatly reducing this battery performance. The diffusive-capacitive of PPy in the reduced state was investigated by EIS (Figure 8). In general, the semicircle in the high to medium frequency region is associated with the charge transfer reaction at the electrolyte/electrode interface. The PPy/AQS/r-GO exhibited a smaller semicircle than that of PPy/AQS at $0 \mathrm{~V}$ vs. SCE (Figure 8A), due to a smaller charge transfer resistance (Rct). This is in agreement with results from CVs. A much larger semicircle was observed for PPy/AQS at $-0.8 \mathrm{~V}$ where PPy is reduced (Figure 8B), and the corresponding Rct is calculated to be $479.3 \Omega$, which increased by 82.9 times compared to its value at $0 \mathrm{~V}$. The Randles equivalent circuit was used to obtain the value of Rct. In contrast, a much smaller Rct was obtained for PPy/AQS/r-GO (136.7 $\Omega$ ). This result could be ascribed to the fact that PPy/AQS/r-GO composite is still conductive even in the reduced state with the presence of reduced graphene oxide, which is consistent with the results obtained for PPy/carbon nanotube (CNT) [12]. This result also supports our conclusions from $\mathrm{CV}$ results, that the presence of r-GO notably enhanced the electrochemical performance of PPy/AQS/r-GO, due to the improved electrical conductivity of reduced PPy facilitating the reaction kinetics of AQS. 

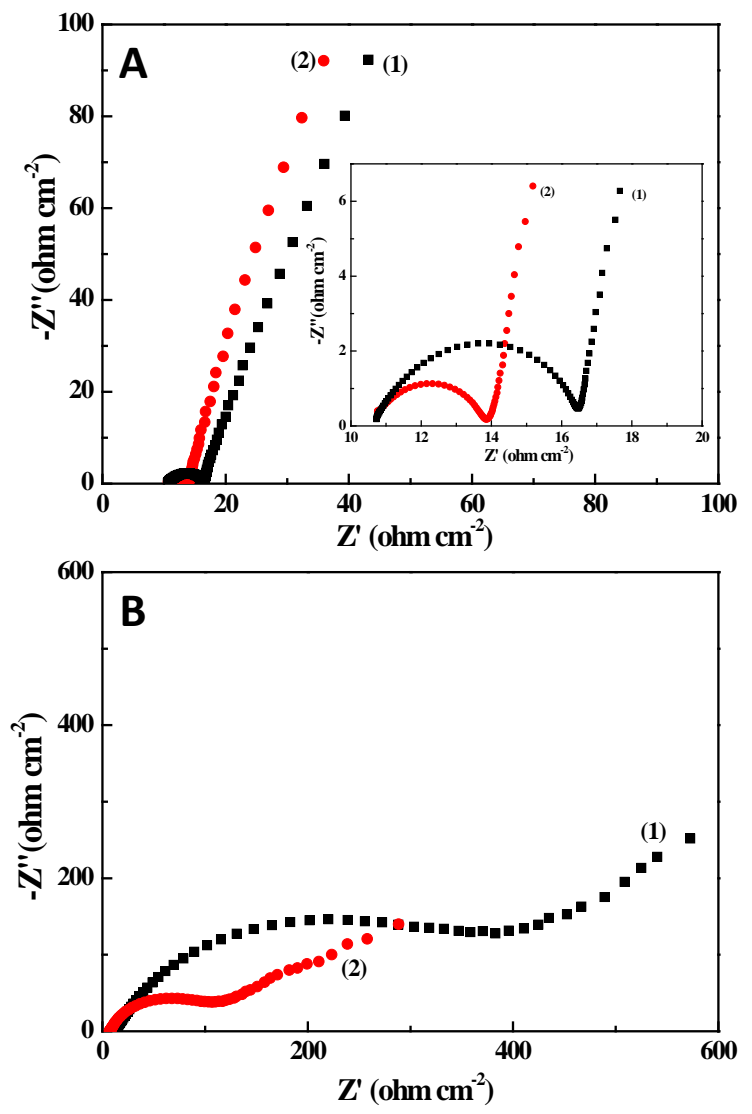

Figure 8: Electrochemical impedance spectra of of PPy/AQS and PPy/AQS/r-GO obtained at $0 \mathrm{~V}$ (A) and -0.8 V (B) vs. SCE in PBS (pH=7.4) solution. (Curve 1, PPy/AQS; Curve 2, PPy/AQS/r-GO; Inset of A, magnified view of the high-frequency region.)

To probe the electrochemical performance of PPy/AQS and PPy/AQS/r-GO further, we investigated the rate capability of the samples in a battery configuration (Figure 9).

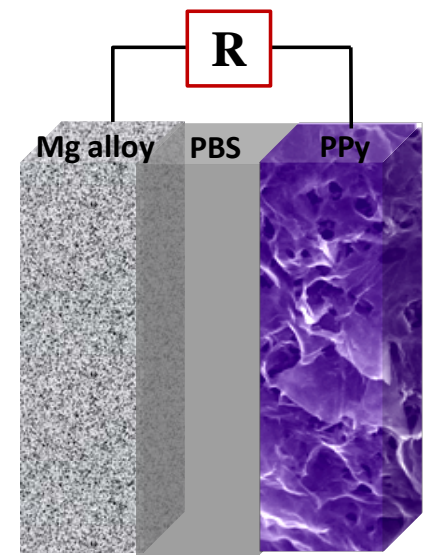

Figure 9: Schematic image of bioelectric battery. 
As expected, PPy/AQS/r-GO showed an exceptionally high rate capability compared with PPy/AQS (Figure 10). At a current density of $25 \mu \mathrm{A} \mathrm{cm}{ }^{-2}$, PPy/AQS/r-GO delivered a voltage of $1.40 \mathrm{~V}$ (plateau potential at the $10^{\text {th }}$ hour), $130 \mathrm{mV}$ higher than that of PPy/AQS (1.27 V). With increasing discharge current, this voltage difference became larger. When the current density increased up to $800 \mu \mathrm{A} \mathrm{cm}{ }^{-2}$, PPy/AQS/r-GO exhibited a voltage of $0.78 \mathrm{~V}$, while only 0.46 V obtained for PPy/AQS. Much lower discharge voltage at higher discharge current for PPy/AQS could be due to the greatly increased resistance of PPy during discharge process. In contrast, the presence of conductive r-GO in PPy/AQS/r-GO matrix could improve the conductivity of the composite electrode, resulting in an enhanced cell performance. It is also noted that PPy/AQS and PPy/AQS/r-GO both delivered a relatively stable discharge plateaus at different current densities, indicative of good stability as cathode for the batteries. The above results clearly demonstrate the enhanced cell performance when using PPy/AQS/r-GO as a cathode material in the bioelectric battery. This can be mainly attributed to two factors: The incorporation of conductive r-GO enhances the conductivity of PPy, in particular at its reduced state, which greatly improved the reaction kinetics of AQS in the matrix. The porous structure facilitates efficient ion and oxygen pathways and also contributes the improved cell performance. 


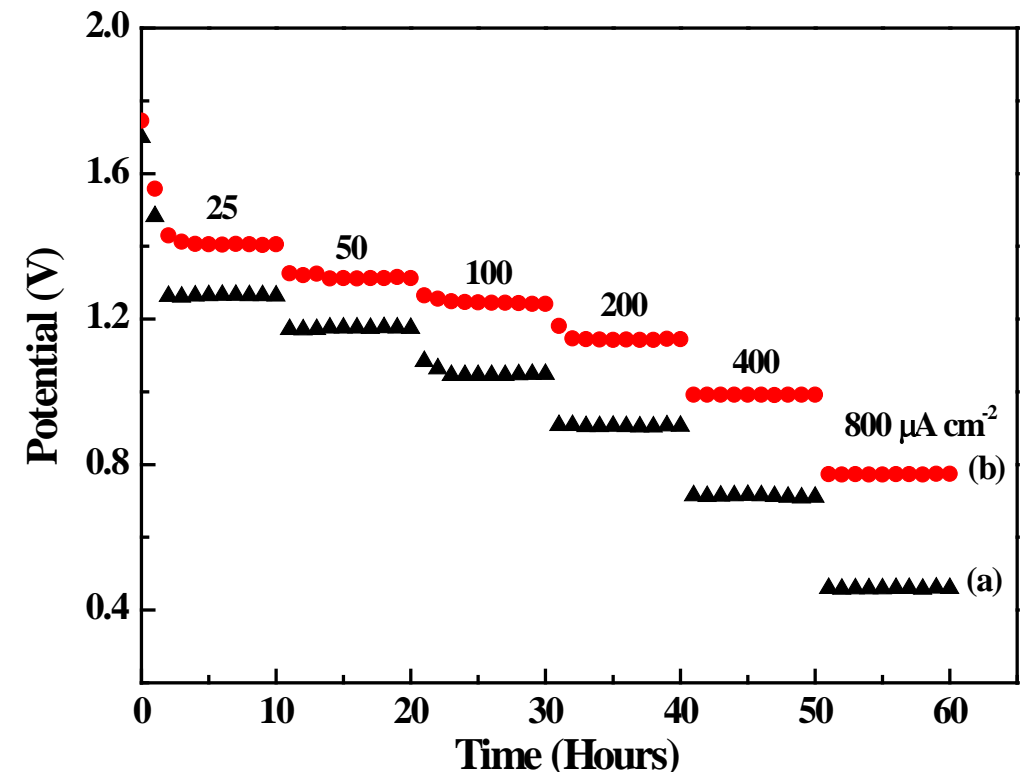

Figure 10: Rate capability of the bioelectric batteries composed of (a) PPy/AQS and (b) PPy/AQS/r-GO cathode at different current densities.

Mg/air system can release an extremely high theoretical specific energy of nearly 6812 Wh $\mathrm{kg}^{-1}$. Its power density becomes a bottleneck for the practical application, particularly in terms of PPy based bioelectric batteries due to the high resistivity of PPy at its reduced state when discharged [30]. The plots of power density vs. current density for PPy composites obtained in this work are shown in Figure 11. The power density is calculated based on the current density and the voltage monitored for each current drain after 10 hours of operation. PPy/pTS was selected for comparison because it had been used in the $\mathrm{Zn}$ or Mg based bioelectric battery [6, 31]. A quasi-linear was found in current density-voltage plot for three electrodes (inset), indicating that the resistance of the cell was primarily dominated by ohmic contributions. It can be concluded that PPy/pTS exhibited the highest resistance as a result of reduction of PPy. $\mathrm{PPy} / \mathrm{pTS}$ almost lost its activity in the cell at the current density of $200 \mu \mathrm{A} \mathrm{cm} \mathrm{cm}^{-2}$ and only displayed a power density of $440 \mathrm{~mW} \mathrm{~m}^{-2}$. In comparison, PPy/AQS and PPy/AQS/r-GO 
delivered a power density of 1812 and $2290 \mathrm{~mW} \mathrm{~m}^{-2}$ under the same current density, respectively. A maximum power density was determined to be $6240.5 \mathrm{~mW} \mathrm{~m}^{-2}$ for PPy/AQS/r-GO at $800 \mu \mathrm{A} \mathrm{cm}{ }^{-2}$. This great improvement in power density moves further the practical application of bioelectric batteries.

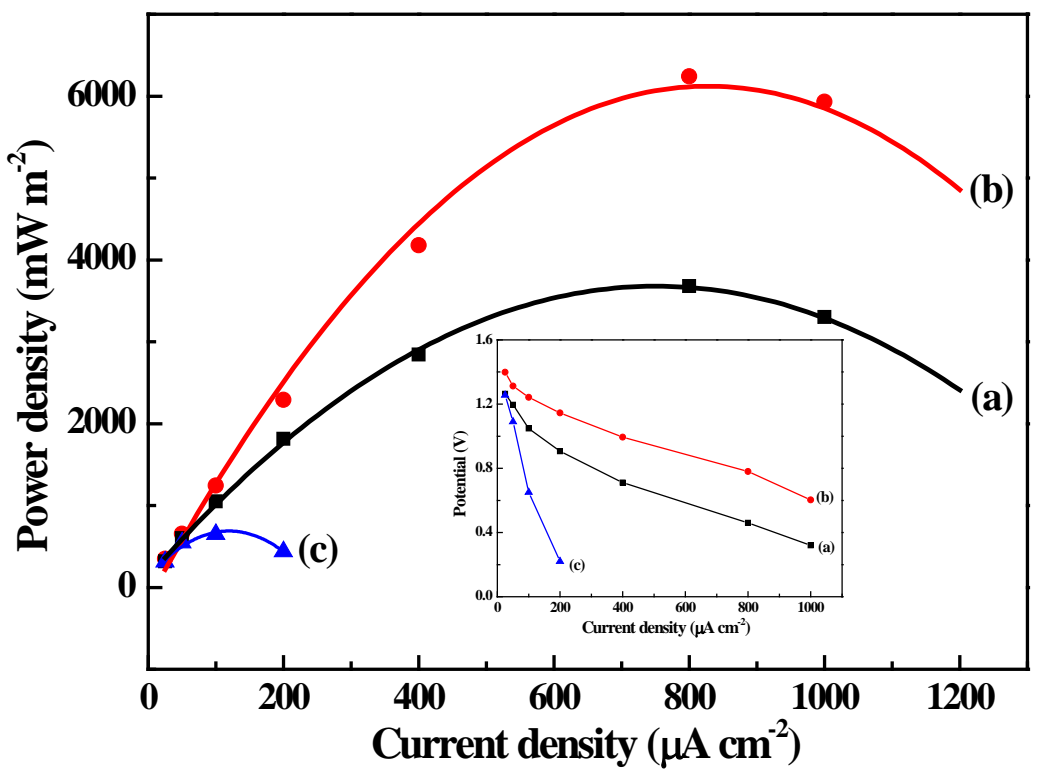

Figure 11: The plots of power density vs. current density for (a) PPy/AQS, (b) PPy/AQS/r-GO and (c) PPy/pTS cathodes in the bioelectric batteries. The insert is rate capabilities of (a) PPy/ AQS, (b) PPy/AQS/r-GO and (c) PPy/pTS cathodes at different current densities.

\section{Conclusions}

PPy/AQS/r-GO composite with porous structure was successfully fabricated via a facile electrochemical route. Such a unique architecture ensures favorable transport kinetics for both ions and oxygen. The presence of conductive r-GO not only provides more favorable electron transport environment for the AQS incorporated in PPy matrix, but also substantially promotes the electrons transport of PPy when it is at its reduced state. These contribute to the 
greatly improved electrocatalytic activity, and a superior cell performance was achieved for the PPy/AQS/r-GO composite with a maximum power density of $6240.5 \mathrm{~mW} \mathrm{~m}^{-2}$. These enhanced electrochemical properties could be further improved by optimizing the electrodeposition parameters. In addition, this simple protocol could be applied broadly by using other anthraquinone sulfonate derivatives, e.g., 9,10-anthraquinone-2,6-disulfonicacidsodiumsalt $\quad$ or 3,4-dihydroxy-9,10-anthraquinone-2-sulfonic acid sodium salt as dopants for PPy. In this PPy/AQS/r-GO composite, PPy and AQS are cytocompatible and the use in biological or medical fields has been demonstrated [32-33]. The cytocompatibility of graphene varies according to its composition and dimensions [34]. In this study graphene was immobilized in polymer matrix due to its large molecule size, which limits the toxic effect from the graphene nanosheet. Moreover, a bioinert $\mathrm{O}_{2}$-permeable, proton-transporting polymer or hydrogel is recommended to encapsulate the bioelectric battery to prevent direct contact with the body fluids [1], which could further increase the safety of the battery.

\section{Acknowledgements}

This work was supported by the Shanghai Committee of Science and Technology under contract No. 14ZR1429500, the International Science \& Technology Cooperation Program of China under contract No. 2012DFG11660 and the National Natural Science Foundation of China under contract No. 21171116. Gordon G. Wallace, Caiyun Wang and Yang Yang also acknowledge the Australian Research Council (ARC) for financial support under the ARC Centre of Excellence for Electromaterials Science. 


\section{References}

[1] Heller A. Potentially implantable miniature batteries. Anal Bioanal Chem 2006; 385(3):469-73

[2] Jiang N, Bornzin GA, Poore JW. Hybrid battery system with bioelectric cell for implantable cardiac therapy device. US Patents 0114236, 2008.

[3] Rodriguez R. Biobattery with nanocrystalline material anode. US Patents 0055570, 2008.

[4] Jiang N, Bornzin GA, Poore JW, Williams S, Yang W, Farazi TG. Bioelectric battery for implantable device application. US Patents 0202105, 2011.

[5] Winther-Jensen B, Gaadingwe M, Macfarlane DR, Forsyth M. Control of magnesium interfacial reactions in aqueous electrolytes towards a biocompatible battery. Electrochim Acta 2008;53(20):5881-4.

[6] Wang C, Zheng W, Yue Z, Too CO, Wallace GG. Buckled, stretchable polypyrrole electrodes for battery applications. Adv Mater 2011;23(31):3580-4.

[7] Kong Y, Wang C, Yang Y, Too CO, Wallace GG. A battery composed of a polypyrrole cathode and a magnesium alloy anode-Toward a bioelectric battery. Synth Met 2012;162(7-8):584-9.

[8] Zhao S, Zhang G, Fu L, Liu L, Fang X, Yang F. Enhanced electrocatalytic performance of anthraquinonemonosulfonate-doped polypyrrole composite: electroanalysis for the specific roles of anthraquinone derivative and polypyrrole layer on oxygen reduction reaction. Electroanal 2011;23(2):355-63. 
[9] Valarselvan S, Manisankar P. Electrocatalytic reduction of oxygen at glassy carbon electrode modified by polypyrrole/anthraquinones composite film in various $\mathrm{pH}$ media. Electrochim Acta 2011;56(20):6945-53.

[10]Zhang G, Yang F. Direct electrochemisty and electrocatalysis of anthraquinone-monosulfonate/ polyaniline hybrid film synthesized by a novel electrochemical doping-dedoping-redoping method on pre-activated spectroscopically pure graphite surface. Phys Chem Chem Phys 2011;13(8):3291-302.

[11]Winther-Jensen B, MacFarlane DR. New generation, metal-free electrocatalysts for fuel cells, solar cells and water splitting. Energy Environ Sci 2011;4(8):2790-8.

[12]Chen GZ, Shaffer MSP, Coleby D, Dixon G, Zhou W, Fray DJ, et al. Carbon nanotube and polypyrrole composites: coating and doping. Adv Mater 2000;12(7):522-6.

[13]Wang J, Xu Y, Chen X, Sun X. Capacitance properties of single wall carbon nanotube/polypyrrole composite films. Compos Sci Technol 2007;67(14):2981-5.

[14]Yoo E, Kim J, Hosono E, Zhou HS, Kudo T, Honma I. Large reversible Li storage of graphene nanosheet families for use in rechargeable lithium ion batteries. Nano Lett 2008;8:2277-82.

[15]Yang X, Zhu J, Qiu L, Li D. Bioinspired effective prevention of restacking in multilayered graphene films: towards the next generation of high-performance supercapacitors. Adv Mater 2011;23(25):2833-8

[16]Zhu Y, Murali S, Cai W, Li X, Suk JW, Potts JR. Graphene and graphene oxide: synthesis, 
properties, and applications. Adv Mater 2010;22(35):3906-24.

[17]Pumera M. Graphene-based nanomaterials for energy storage. Energy Environ Sci 2011;4(3):668-74.

[18]Sun Y, Wu Q, Shi G. Graphene based new energy materials. Energy Environ Sci 2011;4(4):1113-32.

[19]Si P, Ding S, Lou XW, Kim DH. An electrochemically formed three-dimensional structure of polypyrrole/graphene nanoplatelets for high-performance supercapacitors. RSC Advances 2011;1(7):1271-8.

[20]Chang HH, Chang CK, Tsai YC, Liao CS. Electrochemically synthesized graphene/polypyrrole composites and their use in supercapacitor. Carbon 2012;50(6):2331-6.

[21]Yang Y, Wang CY, Yue BB, Gambhir S, Too CO, Wallace GG. Electrochemically synthesized polypyrrole/graphene composite film for lithium batteries. Adv Energy Mater 2012;2(2):266-72.

[22]Jang KS, Lee H, Moon B. Synthesis and characterization of water soluble polypyrrole doped with functional dopants. Synth Met 2004;143(3):289-94.

[23]Cakmak G, Küükyavuz Z, Küükyavuz S. Conductive copolymers of polyaniline, polypyrrole and poly(dimethylsiloxane). Synth Met 2005;151(1):10-8.

[24]Lei J, Liang W, Martin CR. Infrared investigations of pristine, doped and partially doped polypyrrole, Synth Met 1992;48(3):301-12. 
[25]Omastova M, Trchova M, Kovarova J, Stejskal J. Synthesis and structural study of polypyrroles prepared in the presence of surfactants. Synth Met 2003;138(3):447-55.

[26]Liu A, Li C, Bai H, Shi G. Electrochemical deposition of polypyrrole/sulfonated graphene composite films. J Phys Chem C 2010;114(51):22783-9.

[27] Wu J, Wang Y, Zhang D, Hou B. Studies on the electrochemical reduction of oxygen catalyzed by reduced graphene sheets in neutral media. J Power Sources 2011;196(3):1141-4.

[28]Khomenko VG, Barsukov VZ, Katashinskii AS. The catalytic activity of conducting polymers toward oxygen reduction. Electrochim Acta 2005;50(7-8):1675-83.

[29]Lei J, Martin CR. Investigations of the chemical interactions between molecular oxygen and pristine (undoped) polypyrrole. Chem Mater 1995;7(3):578-84.

[30]Cheng F, Chen J. Metal-air batteries: From oxygen reduction electrochemistry to cathode catalysts. Chem Soc Rev 2012;41(6):2172-92.

[31]Li S, Sultana I, Guo Z, Wang C, Wallace GG, Liu HK. Polypyrrole as cathode materials for Zn-polymer battery with various biocompatible aqueous electrolytes. Electrochim Acta 2013;95(0):212-7.

[32]Jackson T, Verrier J, Kochanek P. Anthraquinone-2-sulfonic acid is a novel neurotherapeutic agent. Cell Death Dis 2013;4(1):e451-63.

[33]Wallace GG, Higgins MJ, Moulton SE, Wang C. Nanobionics: the impact of nanotechnology on implantable medical bionic devices. Nanoscale. 2012;4(15):4327-47. 
[34]Feng L, Liu Z. Graphene in biomedicine: opportunities and challenges. Nanomedicine 2011;6(2):317-24. 